\title{
A NATUREZA FRACTAL DE ÁCIDOS HÚMICOS(1)
}

\author{
A. C. SILVA(2), E. S. MENDONÇA ${ }^{(3)}$, M. L. MARTINS ${ }^{(4)}$ \& C. REIS ${ }^{(5)}$
}

\begin{abstract}
RESUMO
Dentre as ferramentas usadas para descrever a estrutura ramificada ou a superfície rugosa e distorci da de ácidos húmi cos (AH), a geometria fractal aparece como uma das mais adequadas para explicar a conformação de partículas húmicas (agregados moleculares). Do ponto de vista experimental, a dimensão fractal (D) de sistemas naturais pode ser determi nada a partir do monitoramento da luz transmitida, não espal hada e não absorvida (turbidimetria ' $\tau$ '). A presença de fractais implica que o sistema pode ser decomposto em partes, em que cada uma, subseqüentemente, é cópia do todo. A determinação do valor ' $D$ ' dessas partículas foi conseguida pela utilização de turbidimetria, em que suspensões de AH-comercial e de AH-E spodossolo foram analisadas por espectrofotometria UV-Vis. $O$ fundamento matemático utilizado foi a lei de potência $\tau \propto \lambda \beta$, em que $\beta<3$ indica a presença de fractal de massa $\left(D_{m}\right) ; 3<\beta \leq 4$ indica fractal de superfície $\left(D_{s}\right)$, e $\beta \cong 3$ indica não-fractal (NF). A declividade das retas ( $\beta$ ) por meio do gráfico $(\log \tau$ vs $\log \lambda)$ permitiu a obtenção de 'D'. Segundo os resultados, partículas de $\mathrm{AH}$ em suspensões aquosas diluídas formam estruturas fractais, cuja geometria pode ser caracterizada por meio de turbidimetria. Entretanto, a faixa de comprimento de onda usada (400 a $550 \mathrm{~nm}$ ) ainda é pequena para se afirmar sobre a natureza fractal de AH e determinar suas dimensões fractais com precisão.
\end{abstract}

Termos de indexação: substâncias húmicas, conformação, turbidimetria.

(1) Recebido para publicação em dezembro de 1999 e aprovado em julho de 2000.

(2) Doutorando em Solos e Nutrição de Plantas, Universidade Federal de Viçosa - UFV. Av. PH Rolfs s/n, 36571-000 Viçosa (MG). E-mail: ale@alunos.ufv.br

(3) Professor de Departamento de Solos - UFV. Bolsista do CNPq. E-mail: esm@alunos.ufv.br

(4) Professor de Departamento de Física - UFV. E-mail: mmartins@mail.ufv.br

(5) Professor de Departamento de Química - UFV. E-mail: cesar@mail.ufv.br 


\title{
SUMMARY: FRACTAL NATURE OF HUMIC ACIDS
}

\begin{abstract}
Among the tools used to describe the ramified structure or the rugged and distorted surface of humic acids (HA), fractal geometry seems to beone of themost adequateto explain the conformation of humic particles (molecul ar aggregates). From theexperimental point of view, fractal dimension (D) of natural systems may be measured through the transmitted light, not scattered or absorved (turbidimetry' $\tau^{\prime}$ ). The presence of fractal s indicates that the system may be decomposed in parts, each part being a copy of the whole. The determination of the fractal dimension (D) of these particles was achieved by theturbidimetric technique, where diluted solutions of commercial-HA and Espodossolo-HA were analyzed by spectrophotometry UV-Vis. Themathematical baseused was the potential law $\tau \propto \lambda^{\beta}$, where $\beta<3$ indicates the presence of mass fractal $\left(D_{m}\right) ; 3<\beta \leq 4$ of surface fractal $\left(D_{s}\right)$ and $\beta \cong 3$, of non-fractal (NF). Theslope of the lines $(\beta)$ was taken from the graphics $(\log \tau$ vs $\log \lambda)$ to obtain D. Theresults show that theH A particles in dil uted aqueous suspensi on form fractals structures, whose geometry may becharacterized by turbidimetry. However, thewavelength range used ( 400 to $550 \mathrm{~nm}$ ) is still small to makestatements about thefractal nature of HA and determine its fractal dimensions with precision.
\end{abstract}

Index terms: humic substances, conformation, turbidimetry.

\section{NTRODUÇÃO}

Nos últimos anos, houve grande interesse na aplicação da teoria fractal em diversos ramos da ciência (Vicsek, 1992; Harrison, 1992; Barton \& La Pointe, 1995). Embora o uso de fractais na ciência do solo seja recente (Burrough, 1983), já existem vários trabalhos nos quais é aplicada a teoria dos fractais no estudo estrutural de substâncias húmicas (Rice \& Lin, 1994; Österberg \& Mortensen, 1994; Senesi, et al., 1997).

A fração humificada do solo, chamada de substâncias húmicas, inclui os ácidos húmicos $(\mathrm{AH})$, os ácidos fúlvicos (AF) e humina $(H)$. Dada a facilidade de extraçãoe presença, preferencialmente, nas camadas superficiais dos solos onde a concentração de AH é maior que a de AF (Lobartini et al., 1991), os AHs são uma das frações mais estudadas.

Os AHs são macromoléculas heterogêneas formadas por decomposição biológica de plantas e animais (Aiken, et al.,1985). São caracterizados por ausência de repetição estrutural, desordem molecular (Hayes et al., 1989) e por larga variedade de tamanhos e formas, que implicam uma porosidade/compactação variável, assumindo forma sólida ou estado coloidal (Swift, 1989). Tais características influem no número, tipo e disponibilidade de sítios reativos, na capacidade de adsorção e na extensa interação de minerais e íons metálicos (Stevenson, 1994). Dentre as ferramentas usadas para descrever a estrutura ramificada ou a superfície rugosa e distorcida de $\mathrm{AH}$, a geometria fractal aparece como uma das mais adequadas para explicar a conformação de partículas húmicas (agregados moleculares).
O termo "fractal" foi introduzido na década de 70 pel o matemático Benôit Mandel brot, para designar objetos e estruturas complexas dotadas da propriedade de auto-similaridade (Chaves, 1989). Estruturas auto-similares apresentam detal hes (ramificações, poros ou rugosidades) em certa faixa de escala de comprimento, cuja forma é a mesma em diferentes escalas. Assim, se parte da estrutura for ampliada terá a mesma forma do todo (Mandel brot, 1983).

A maior parte dos fenômenos naturais apresenta comportamento não-linear, capaz de gerar padrões bastante complexos para serem adequadamente descritos pela geometria euclidiana (Pires \& Costa, 1992). U ma alternativa que minimiza as limitações presentes na descrição clássica (euclidiana) dos fenômenos irregulares da natureza é a geometria fractal, que, ao contrário da euclidiana, nãose baseia em objetos regulares, suaves e diferenciáveis (Guzman et al., 1993). Cabe ressaltar que os conjuntos na geometria eucli idiana são descritos por equações al gébricas, enquanto a geometria fractal é descrita por algoritmos recursivos. Conhecendo o "motivo" gerador de determinada estrutura, podese, a partir daí, obter a estrutura completa (Vicsek, 1992).

Acredita-se que o aspecto mais básico de um objeto fractal seja a sua dimensão. U m modo simples eintuitivo de associar uma dimensão a determinado conjunto é contar o número mínimo $N(\varepsilon)$ de quadrículas, de lado $(\varepsilon)$, necessárias para cobrir completamente o conjunto. Esse número obedece a uma lei de potência: $N(\varepsilon)=A \cdot \varepsilon^{-D}$, que definee permite calcular o expoente $D$, queéa dimensão de cobertura ou, mais simplesmente, a dimensão fractal (Mandel brot, 1983). 
A dimensão fractal (D) pode apresentar, ao contrário da dimensão euclidiana (d), valores fracionários que dependem do sistema: $1<D<2$, para uma curva; $2<D<3$, para superfície, e $2<\mathrm{D}<3$, para um 'sólido'. E sse val or éuma medida da proporção do espaço real mente ocupado por um sistema desordenado, podendo refletir a conformação das partículas que o constituem (Harrison, 1992).

Do ponto de vista experimental, a dimensão fractal de sistemas naturais pode ser determinada a partir da análise de imagens, medidas de adsor ção defluidos, espal hamento deluz, etc. A turbidimetria $(\tau)$ baseada no monitoramento da luz transmitida (não espalhada e não absorvida) apresenta-se, em comparação com as demais técnicas, como uma boa alternativa no estudo de fractais em suspensões diluídas (Horne, 1987).

Neste sentido, pretende-se, por meio desta técnica, confirmar a natureza fractal de partículas húmicas em suspensão aquosa, determinar as dimensões fractais associadas e investigar as possíveis mudanças conformacionais e regimes de agregação dessas partículas, considerando o pH e o tempo de agitação da suspensão.

$\mathrm{Na}$ geometria fractal, a invariância de escala ou a auto-similaridade é caracterizada por uma lei de potência da forma $p \propto v^{\Upsilon}$, em que $p=$ propriedade, $v$ = variável e $\Upsilon$ =expoente, que pode ser relacionado com a dimensão fractal (Senesi, 1994). Essa expressão garante que, sobre uma faixa de valores da escala da variável independente $v$, de pel o menos uma ordem de grandeza, o expoente $\Upsilon$ (e, portanto, o val or de D) pode ser cal culado diretamente dos dados experimentais a partir da inclinação da reta do gráfico log p vs log v. U ma dessas propriedades é a própria massa do objeto. Para qual quer conjunto de pontos, é possível, a partir de seu centro de massa, traçar círculos sucessivos de raio r (circunferência, para $d=2$; esferas, para $d=3$ ) e determinar como o número de pontos (a massa) do conjunto contidos nesses círculos al tera com a variação do raio r. Para qualquer conjunto, obter-se-á M. $(r) \propto r^{D}$ (Vicsek, 1992).

Nos objetos eucl idianos (não-fractais), encontrarse-ão valores inteiros $(D=d)$. J á nos conjuntos fractais, serão encontrados, geralmente, valores fracionários $(\mathrm{D}<\mathrm{d})$. Tais conjuntos com $\mathrm{D}<\mathrm{d}$ podem ser objetos ramificados ou porosos. As estruturas fractais ramificadas (como uma árvore) e porosas (como uma esponja) são ditas fractais de massa $\left(D_{m}\right)$ e de poros $\left(D_{p}\right)$, respectivamente. Por outro lado, existem conjuntos cujos interiores são compactos ( $D=d$ e, portanto, $M \propto r^{d}$ ) eapenas suas superfícies são fragmentadas e irregulares, caso em que são ditos fractais de superfície $\left(D_{s}\right)$.

A lei de potência: $\tau \propto \lambda^{\beta}$ (em que $\tau$ é a turbidez, $\lambda$ é o comprimento de onda e $\beta$ é um expoente diretamente relacionado com $D$ ) é observada em sistemas de partículas em suspensão diluída, conforme demonstrado pelo modelo matemático descrito por Horne (1987). A turbidimetria é definida pela equação: $\tau=$ d.In.(Io/I), em que d é o comprimento óptico, lo e I são as intensidades (incidente e transmitida), respectivamente (Skoog, et al., 1992).

Na turbidimetria, o expoente $\beta$ indica a natureza do sistema: se $\beta \neq 3$, o sistema é desordenado, tem natureza fractal; se $\beta=3$, o sistema é ordenado e não é fractal (Senesi, et al., 1997). Medidas de turbidimetria (Senesi et al., 1996), espal hamento de neutrons (Österberg \& Mortensen, 1994), espalhamento de raios-x (Rice \& Lin, 1993), dentre outras, já foram aplicadas no estudo da conformação e fenômenos de agregação de partículas de $\mathrm{AH}$.

Em geral, sistemas húmicos não são considerados estruturas porosas, pois o tamanho de seus poros (da ordem de nanômetros) é muito pequeno, quando comparado com a escala da superfície dessas partículas. Comisso, as partículas deAH apresentamse como fractais de massa $\left(D_{m}\right)$ ou de superfície $\left(D_{\mathrm{s}}\right)$. A caracterização de $A H$ pela dimensão fractal $D_{m}$ ou $D_{s}$ é realizada pela determinação do expoente $\beta$, a partir da inclinação da parte linear dos pontos do gráfico $\log \tau$ vs $\log \lambda$. Se $\beta<3$, então o $A H$ será um fractal de massa, neste caso $D_{m}=\beta$; se $3<\beta \leq 4$, então o AH será um fractal de superfície, neste caso $D_{s}=6$ - $\beta$; finalmente, se $\beta \cong 3$, o $A H$ não terá natureza fractal, neste caso $D=d$ (Senesi, 1994).

\section{MATERIAL E MÉTODOS}

Foram estudadas duas amostras de $\mathrm{AH}$ (solo e comercial). Os AHs extraídos de solo ( $\mathrm{AH}$ espodossolo) foram obtidos de uma amostra de Espodossolo (camada de 0-50 cm), col etada em Lavras Novas (MG). O procedimento de extração obedeceu às normas da IHSS, Sociedade Internacional deSubstâncias Húmicas (Swift, 1996). A purificação foi realizada somente por meio de lavagens sucessivas com sol ução de $\mathrm{HCl}-\mathrm{HF}$ a 0,5\%. Para oteste da presença de cloretos $\left(\mathrm{Cl}^{-}\right)$, utilizou-se $\mathrm{AgNO}_{3}$. Após testenegativo, os AHs foramliofilizados e estocados. As amostras do AH-comercial foram adquiridas comercialmente pela F ukla S.A.

Os teores de carbono, hidrogênio e nitrogênio nas amostras foram obtidos via anal isador de elementos CNHS (Perkin Elmer, PE-2400). A razão entre as absorvâncias a 465 e 665 nm (conhecida como razão $E_{4} / E_{6}$ ) foi obtida segundo método descrito por Chen et al. (1977). Os espectros de infravermel ho das amostras foram lidos na faixa de $4.000-500 \mathrm{~cm}^{-1} \mathrm{em}$ pastilhas de $\mathrm{KBr}$, utilizando um espectrômetro IV (Perkin Elmer Spectrum 1000).

Suspensões diluídas ( $30 \mathrm{mg} \mathrm{L}^{-1}$ ) das duas amostras de $\mathrm{AH}$ foram preparadas em água deionizada. Para evitar problemas na leitura turbidimétrica 
decorrente do efeito de espalhamento múltiplo e interação de partículas, requereu-se o uso de suspensões diluídas. $\mathrm{O}$ pH das suspensões foi ajustado para 3,0; 5,0 e 7,0 pela adição de $\mathrm{NaOH}$ e $\mathrm{HCl}$, na mesma força iônica. As suspensões foram, então, equilibradas a $25^{\circ} \mathrm{C}$, por $1,3,8$ e $12 \mathrm{~h}$, por meio de agitador horizontal .

A turbidez $(\tau)$ das suspensões foi medida em espectrofotômetro UV-Vis (Hitachi U2000), utilizando célula de quartzo de $1 \mathrm{~cm}$ de percurso ótico. A coleta de dados foi automatizada por meio de interface entre o aparel ho e um computador. As medidas de ' $\tau$ ' em função de ' $\lambda$ ' foram, primeiramente, realizadas em varredura de 400 a $800 \mathrm{~nm}$. Em razão da falta de linearidade para valores de ' $\lambda$ ' acima de $550 \mathrm{~nm}$, somente a faixa de $400-550 \mathrm{~nm}$ (interval os de $1 \mathrm{~nm}$ ) foi considerada.

\section{RESULTADOSE DISCUSSÃO}

Os teores de carbono, hidrogênio e nitrogênio nas amostras foram de 50,8; 3,51 e1,75\%, respectivamente, para amostra de $\mathrm{AH}$-E spodossolo e de 43,1; 4,87 e $0,67 \%$, para AH-comercial. A razão N/C obtida $(0,035)$ para $\mathrm{AH}$-E spodossolo está coerente com as obtidas por Rice \& Macharty (1991), citados por Spark et al. (1997), para AHs extraídos de solos. J á a razão N/C $(0,015)$ para $\mathrm{AH}$-comercial enquadrou-se na faixa de AHs extraídos de carvão. $O$ valor da razão $E_{4} / E_{6}$ (comumente usada como indicativo do grau de humificação) foi de 3,60 e 3,37 para AH-Espodossolo e $\mathrm{AH}$-comercial, respectivamente. Esses val ores estão dentro da faixa de $\mathrm{AH}$, que devem ser menores que 5,0 (Stevenson, 1994). O teor de cinzas foi de 8 e $14 \%$, para $\mathrm{AH}$-EspodossoloeAH-comercial, respectivamente.

O espectro no infravermel ho de $\mathrm{AH}-\mathrm{E}$ spodossolo (Figura 1a) demonstrou uma feição muito similar à obtida por Benites et al. (1999) para AHs extraídos e purificados de horizonte Bhs de um Espodossolo col etado em Araponga (MG), excetuando a presença da banda $532 \mathrm{~cm}^{-1}$ (deformação angular de O-H em gibbsita ou caulinita). A presença de impurezas é explicada, possivelmente, por diferenças na purificação. J á o espectro deAH-comercial (Figura 1b) demonstrou-se similar ao obtido por MacCarthy \& Rice (1985).

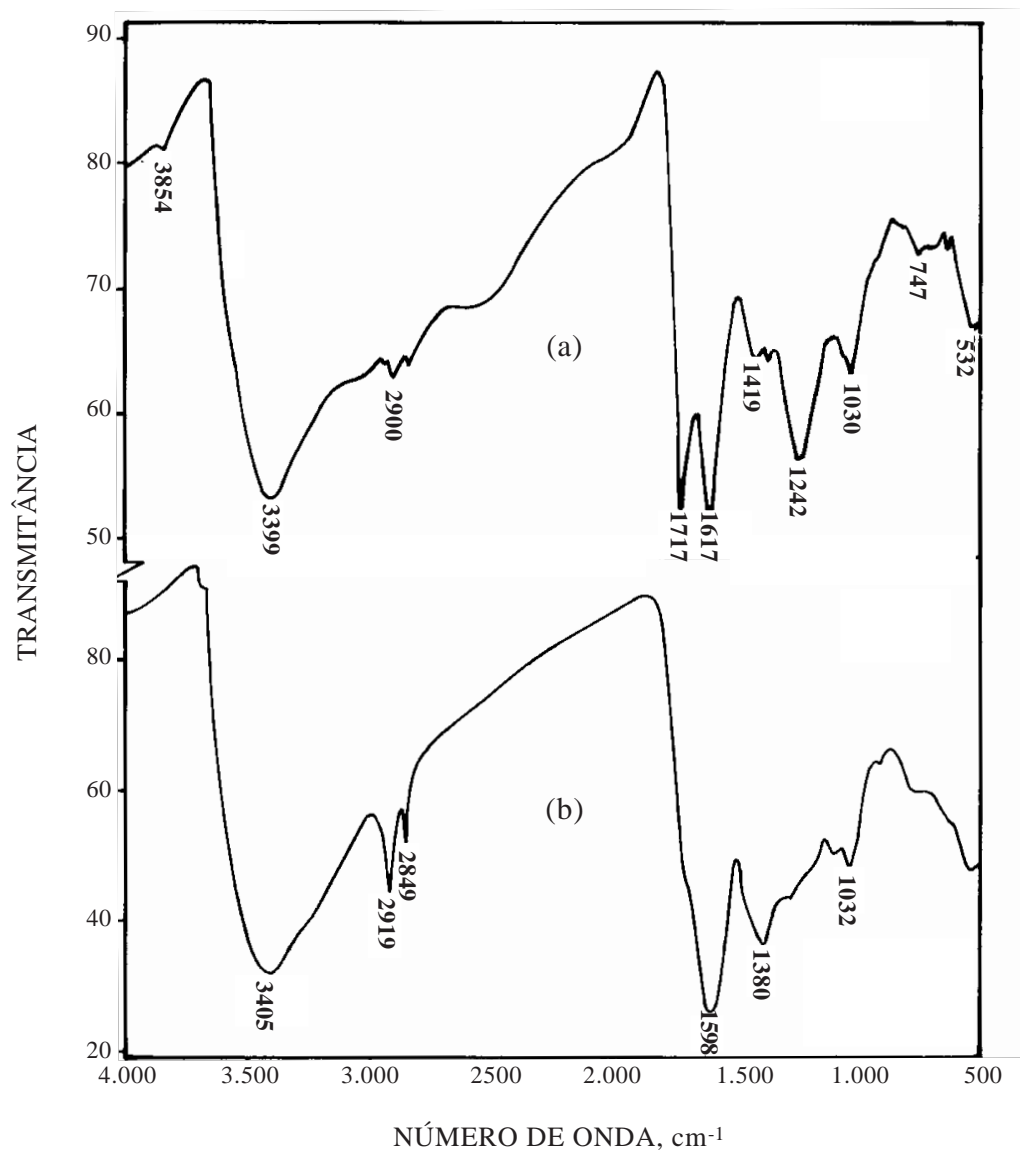

Figura 1. Espectros de infravermelho de AH-E spodossolo (a) e AH-comercial (b). 
A dependência em lei de potência de $\tau$ em função de $\lambda$ foi confirmada para todas as suspensões a diferentes val ores de pH $(3,0 ; 5,0$ e 7,0$)$ e tempos de agitação ( $1,3,8,12 \mathrm{~h})$, tendo sido a linearidade dos pontos experimentais (observada no gráfico do $\log \tau$ em função do $\log \lambda$ ) considerada satisfatória $(R \geq 0,996)$. Assim, as partículas em estudo podem exibir estrutura fractal de massa ou de superfície, enquanto os valores de $D_{m}$ e $D_{s}$ podem ser obtidos diretamente de ' $\beta$ ' (Senesi et al., 1997). Como ilustração, dois exemplos (pH 3,0 em diferentes tempos de agitação e tempo de $1 \mathrm{~h}$ em diferentes val ores depH) foram apresentados na figura 2. Podese observar pequena variação da declividade das retas ( $\beta)$, conformeotempo deagitação. No AH-E spodossolo, ocorreu cruzamento de linhas em torno de $\log \lambda=-6,31$, ou seja, duas inclinações $\beta$ (e, portanto, dois valores distintos de 'D'): uma para $\log \lambda<-6,31$ e outra maior para $\log \lambda>-6,31$. Esse cruzamento pode significar a existência de estruturas distintas nestas duas faixas de escala de comprimento.
Embora as macromoléculas húmicas, no estado col oidal , sofram modificação (por ionização de grupos ou formação de pontes de hidrogênio) de acordo com opH do meio (Swift, 1989), nãofoi observada variação nos valores de pH 5,0 e 7,0 para ambas as amostras (Quadros 1 e2). Os tempos de agitação (1, 3, 8 e 12 h) não influenciaram os valores da dimensão fractal para AH-Espodossol o nos valores de pH 3,0 e 7,0.

Percebeu-se no AH-Espodossolo uma transição morfológica em sua estrutura que passou de fractal de massa $(\mathrm{pH} \mathrm{3,0)}$ para fractal de superfície ( $\mathrm{pH} \geq 5,0$ ) (Quadro 1). As partículas húmicas de Espodossolo, em suspensão, evoluíram de estruturas ramificadas, fragmentadas e menos compactas para estruturas compactas com superfícies irregulares ou rugosas. Esse fato pode estar relacionado com a fragmentação crescente das estruturas ramificadas com o aumento do pH. Fragmentadas, essas partículas seriam menores, quase desprovidas de pontes ou ramos e, portanto, poder-se-iam agregar num empacotamento mais compacto.
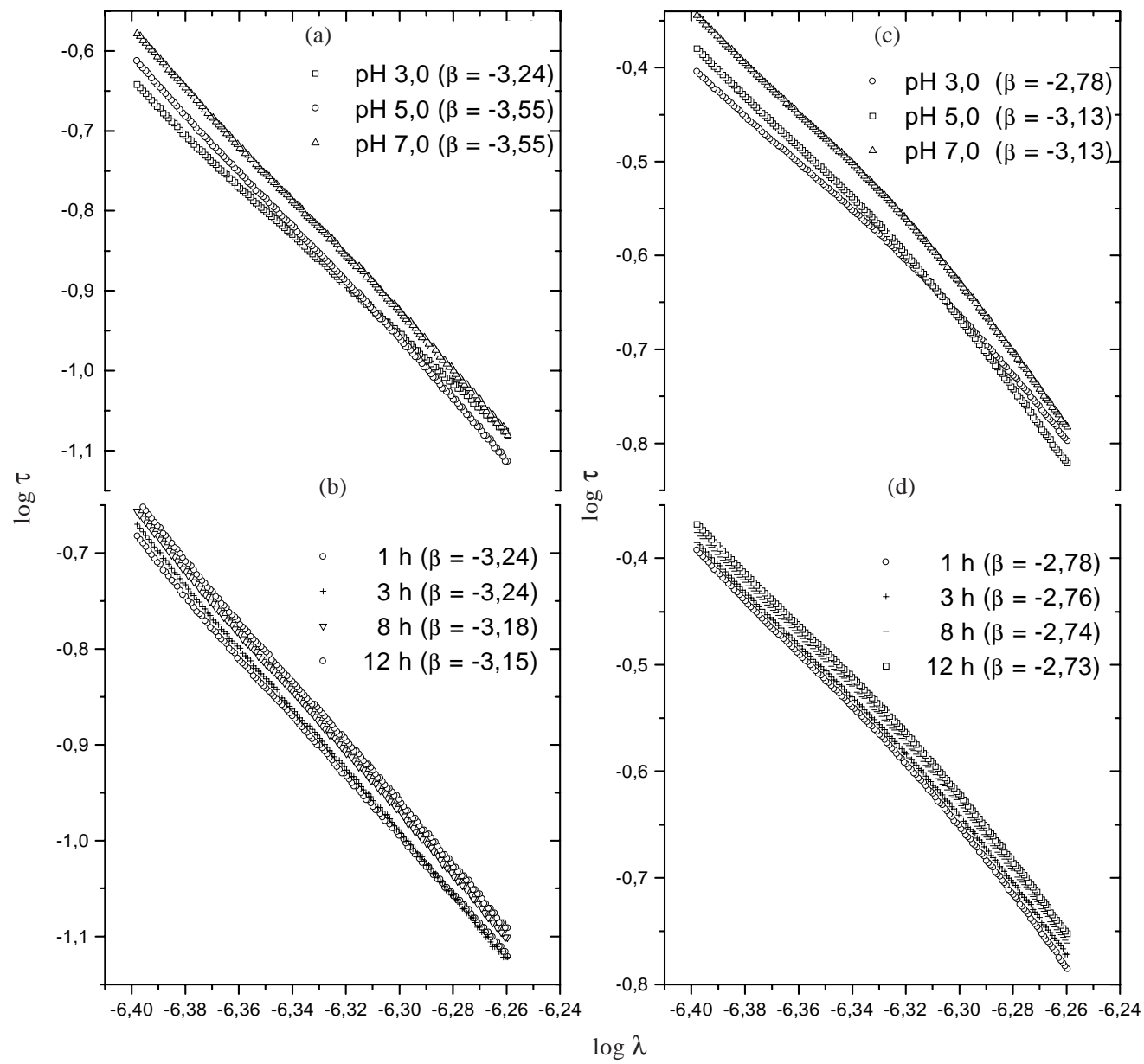

Figura 2. Comportamento da linearização das retas do gráfico $\log \tau$ vs $\log \lambda$ para suspensões aquosas de AH-comercial com tempo de agitação de $1 \mathrm{~h}$, em diferentes valores de pH (a), em pH 3,0, para diferentes tempos de agitação(b), e de AH-E spodossolo com tempo de $1 \mathrm{~h}$, em diferentes valores de pH (c), em pH 3,0, para diferentes tempos de agitação(d). 
Esse comportamento contraria diretamente a tendência de desagregação crescente de $\mathrm{AH}$ observada para valores de pH entre 3,5 e 7,0 (Wershaw \& Pinckney,1973, citados por Wershaw (1989). Esse resultado mostrou-se contrário aos observados por Senesi et al. (1996), que utilizaram AH padrões, adquiridos comercial mente pela I HSS (teor de cinzas abaixo de 1\%). Essa situação pode estar relacionada com o tipo de amostra de AH-Espodossolo, pois é sabido que mudanças conformacionais dependem das características (composição e estrutura) do AH em estudo, e estas, por sua vez, estão diretamente relacionadas com sua origem, processo de extração e condições do meio (ex. força iônica).

O valor de D em função do pH e do tempo, para cada amostra, foi determinado a partir de um ajuste não-linear (quadrático). As funções encontradas foram apresentadas por meio de equação de suas respectivas superfícies de resposta:

$\mathrm{D}=2,4052+0,1587^{* *} \mathrm{pH}-0,01282^{* *} \mathrm{pH}^{2}-0,0014^{* *} \mathrm{~T}$ $\left(R^{2}=0,96\right)$, para $A H-E$ spodossolo, e

$\mathrm{D}=3,8126-0,4662^{* *} \mathrm{pH}+0,03875^{* *} \mathrm{pH}^{2}+0,0058^{* *} \mathrm{~T}$ $\left(R^{2}=0,94\right)$, para $A H$-comercial, onde $T=$ tempo em horas.

A equação obtida para $\mathrm{AH}$-E spodossolo deve ser ol hada com cautela, uma vez que considera somente o valor da dimensão fractal (D), independentemente de sua natureza, ou seja, de ser $D_{m}$ ou $D_{s}$. Senesi et al.(1994) observaram, em suspensões de AHs extraídos deUltissolo, diminuição no valor de D com o aumento do pH. Essa tendência só foi confirmada para o $\mathrm{AH}$-comercial entre os valores de $\mathrm{pH} 3,0 \mathrm{e}$ 5,0 em todos os tempos de agitação. Para o AHEspodossol o esta tendência não foi confirmada. Essa diferença pode ser devida à natureza das amostras e mudanças conformacionais em uma mesma amostra. Wershaw \& Pinckney, 1973, citados por Wershaw (1989), relataram três modificações no comportamento de $\mathrm{AH}$ : (a) aumento da agregação a $\mathrm{pH}<3,5$ com pequena desagr egação a $\mathrm{pH}>3,5$; (b) desagregação a $\mathrm{pH} \leq 7,0$ e reagregação a $\mathrm{pH} 7,0$, e (c) contínua diminuição da reagregação com o aumento do $\mathrm{pH}>7,0$.

Rice \& Lin (1994) demonstraram que os AHs podem ser descritos pela geometria fractal, os valores de $D$ encontrados para amostras de sedimento de rio foram 2,2 para $\mathrm{AH}$, indicando que os $\mathrm{AHs}$, nesse caso, apresentaram estruturas levementeaplainadas. Österberg \& Mortensen (1992) aplicaram, por meio de espalhamento de neutrons, a teoria fractal ao estudo de duas amostras de $\mathrm{AH}$, uma extraída de solo e outra comprada comercialmente (IHSS) e obtiveram valores de 2,3 $\pm 0,1$ para ambas as amostras, indicando que as partículas de AH estavam mais fechadas.

Quanto à influência do tempo de agitação, os resultados não foram coerentes com os obtidos por Senesi et al. (1996), cujos valores de D diminuíram com o aumento da agitação. A explicação pode ser

\section{Quadro 1. Valores da dimensão fractal de suspensões de AH-Espodossolo}

\begin{tabular}{ccccc}
\hline \multirow{2}{*}{ Valor de $\mathbf{p H}$} & \multicolumn{4}{c}{ Tempo de agitação (h) } \\
\cline { 2 - 5 } & $\mathbf{1}$ & $\mathbf{3}$ & $\mathbf{8}$ & $\mathbf{1 2}$ \\
\hline 3,0 & $\mathrm{D}_{\mathrm{m}}=2,78 \pm 0,01$ & $\mathrm{D}_{\mathrm{m}}=2,76 \pm 0,01$ & $\mathrm{D}_{\mathrm{m}}=2,74 \pm 0,01$ & $\mathrm{D}_{\mathrm{m}}=2,73 \pm 0,01$ \\
5,0 & $\mathrm{D}_{\mathrm{s}}=2,87 \pm 0,02$ & $\mathrm{D}_{\mathrm{s}}=2,86 \pm 0,03$ & $\mathrm{D}_{\mathrm{s}}=2,87 \pm 0,03$ & $\mathrm{D}_{\mathrm{s}}=2,85 \pm 0,04$ \\
7,0 & $\mathrm{D}_{\mathrm{s}}=2,87 \pm 0,03$ & $\mathrm{D}_{\mathrm{s}}=2,87 \pm 0,04$ & $\mathrm{D}_{\mathrm{s}}=2,88 \pm 0,04$ & $\mathrm{D}_{\mathrm{s}}=2,86 \pm 0,04$ \\
\hline
\end{tabular}

$\mathrm{D}_{\mathrm{m}}=$ Dimensão fractal de massa, $\mathrm{D}_{\mathrm{s}}=$ Dimensão fractal de superfície.

Quadro 2. Valores da dimensão fractal de suspensões de AH-Comercial

\begin{tabular}{ccccc}
\hline \multirow{2}{*}{ Valor de $\mathbf{p H}$} & \multicolumn{4}{c}{ Tempo de agitação $(\mathbf{h})$} \\
\cline { 2 - 5 } & $\mathbf{1}$ & $\mathbf{3}$ & $\mathbf{8}$ & $\mathbf{1 2}$ \\
\hline 3,0 & $\mathrm{D}_{\mathrm{s}}=2,76 \pm 0,02$ & $\mathrm{D}_{\mathrm{s}}=2,76 \pm 0,01$ & $\mathrm{D}_{\mathrm{s}}=2,82 \pm 0,04$ & $\mathrm{D}_{\mathrm{s}}=2,85 \pm 0,04$ \\
5,0 & $\mathrm{D}_{\mathrm{s}}=2,45 \pm 0,01$ & $\mathrm{D}_{\mathrm{s}}=2,49 \pm 0,01$ & $\mathrm{D}_{\mathrm{s}}=2,50 \pm 0,01$ & $D_{\mathrm{s}}=2,50 \pm 0,01$ \\
7,0 & $\mathrm{D}_{\mathrm{s}}=2,45 \pm 0,01$ & $\mathrm{D}_{\mathrm{s}}=2,48 \pm 0,02$ & $\mathrm{D}_{\mathrm{s}}=2,49 \pm 0,01$ & $D_{\mathrm{s}}=2,51 \pm 0,05$ \\
\hline
\end{tabular}

$\mathrm{D}_{\mathrm{s}}=$ Dimensão fractal de superfície. 
devida à diferença de contato entre as partículas, pois esses autores utilizaram agitação magnética, que, em geral, fornece mai or vel ocidade/contato em relação à agitação horizontal. E mbora Österberg et al. (1994) tenham usado agitação magnética, seus resultados também diferiram daquel es de Senesi et al. (1996). Contudo, aqueles autores trabalharam com força iônica maior $(0,1 \mathrm{M}$ de $\mathrm{NaCl})$ e maior concentração de AH $\left(3 \mathrm{~g} \mathrm{~L}^{-1}\right)$. Segundo Ghosh \& Schnitzer (1980), quanto maiores a força iônica e a concentração da suspensão, maior a tendência de formação de pontes de hidrogênio; logo, mais enovelada estará a molécula de $\mathrm{AH}$, levando a um aumento no valor de D.

\section{CONCLUSÕES}

1. Partículas deAH em suspensão aquosa diluída (tanto extraídas de solo quanto adquiridas comercialmente) formam estruturas fractais, cuja geometria, ou dimensãofractal, podeser caracterizada por meio deturbidimetria. Entretanto, éimportante ressaltar quea faixa de comprimento de onda usada (400 a $550 \mathrm{~nm}$ ) ainda é pequena para se afirmar, com certeza, sobre a natureza fractal dos AHs e determinar suas dimensões fractais com precisão.

2. Partículas de AH-E spodossol o demonstraram uma transição morfol ógica confirmada pela evolução de estruturas fractais de massa (menos compacta) para fractais de superfície (mais compacta) com o aumento da suspensão do pH. E ssa evolução, diferentementedo AH-comercial, foi oposta à observada por Senesi et al. (1996) e reforçou ainda mais a idéia de que o comportamento fractal de $\mathrm{AH}$ dependeu fortemente de sua origem e processo de extração.

3. A presença deinclinações ' $\beta$ ' (dimensão fractal) distintas, para faixas de comprimento de onda diferentes, podeindicar que os AHs sejam estruturas multifractais.

4. Para melhor compreensão da aplicabilidade dessa ferramenta (dimensão fractal) em estudos de $\mathrm{AH}$ recomendam-se pesquisas adicionais que visem comparar os resultados efornecer informações sobre aspectos dinâmicos de agregação das partículas de $\mathrm{AH}$, objetivando revelar as características físicoquímicas que controlam a natureza fractal dessas partículas.

\section{LITERATURA CITADA}

AIKEN, G.R.; MCKNIGHT, D.M.; WERSHAW, R.L. \& MacCARTHY, P. An introduction tohumic substances in soil, sediment, and water. In: AIKEN, G.R., MCKNIGHT, D.M. \& WE RSHAW, R.L., eds. Humic substances in soil, sediment and water: geochemistry, isolation and characterization. New York, J ohn Wiley \& Sons, 1985. 692p.
BARTON, C.C. \& LA PONTE, P.R. Fractais in the earth sciences. New York, Plennum Press, 1995. 265p.

BENITES, V.M.; MENDONÇA, E.S.; SCHAEFER, C.E.R. \& MARTIN-NETO, L. Caracterização dos ácidos húmicos extraídos de um Latossolo Vermelho-Amarelo e de um Podzol por análise termodiferencial e pela espectroscopia de absorção no infravermelho. R. Bras. Ci. Solo, 23:543551, 1999.

BURROUGH, P.A. Multiscale source of spacial variation in soil. The aplication of fractal concepts to nested levels of soil variation. J. Soil. Sci., 34:577-597, 1983.

CHAVES, C.M.G.F. Fenômenos de agregação. Ci. Hoje, 10:27-32, 1989.

CHEN, Y.; SENESI, N.\& SCHNITZER, M. Information provided on humic substances by E4/E 6 ratios. Soil Sci. Soc. Am. J ., 41:352-358, 1977.

GHOSH, K. \& SCHNITZER, M. Macromolecular structures of humic substances. Soil Sci. 129:266-276, 1980.

GUZMAN.; M.; MARTIN, M.A.; MORÁN, M. \& REYES, M. Estructuras fractales y sus aplicaciones. Barcel ona, Labor, 1993. 279p.

HARRISON, A. Fractals in chemistry. Oxford, Science Publications, 1992. 90p.

HAYES, M.H.B.; MaCARTHY, P.; MALCOLM, R.L. \& SWIFT, R.S. Structures of humic substances: the emergence of forms (3-31). In: HAYES, M.H.B.; MaCARTHY, P.; MALCOLM, R.L. \& SWIFT, R.S. Humic Substance II: In search of structure: setting the scene. New York, J ohn Wiley \& Sons, 1989. p.??

HORNE, D.S. Determination of the fractal dimension using turbidimetric techniques. Faraday Discuss. Chem. Soc., 83:259-270, 1987.

LOBARTINI, J.C.; TAN, K.H.; ASMUSSEN, L.E.; LEONARD, R.A.; HIMMELSBACH, D. \& GINGLE, A.R. Chemical and spectral diferences in humic matter from swamps, streams and soils in the southeastern United States. Geoderma, 49:241-254, 1991.

MacCARTHY, P. \& RICE, J. Spectroscopic methods for determining functionality in humic substances. In: AIKEN, G.R., MCKNIGHT, D.M.; WERSHAW, R.L. \& MacCARTHY, P. Humic substances in soil, sediment, and water. New York, J ohn Wiley \& Sons, 1985. p.527-559.

MANDELBROT, B.B. The fractal geometry of nature - update and augmented. New York, F reeman, 1983. 568p.

ÖSTERBERG, R. \& MORTENSEN, K. Fractal dimension of humic acids. Eur. Biophys J ., 21:163-167, 1992.

ÖSTERBERG, R.\& MORTENSEN, K. Fractal geometry of humic acids, temperature-dependent restructuring studied by small-angle neutron scattering. In: SENESI, N.\& MIANO, T.M. Humic substances in the global environment and implications on human health. Amsterdam, Elsevier Science, 1994. p.127-132.

ÖSTERBERG, R.; SZAJ ADAK, L. \& MORTENSEN, K. Temperature-dependent restruring of fractal humic acids: A proton-dependent process. Environ. Int., 20:77-88, 1994.

PIRES, A.S.T. \& COSTA, B.V.A. A desordem inevitável. Ci. Hoje, 14:35-39, 1992 
RICE, J .A. \& LIN, J .S. Fractal dimensions of humic materials. In: SENESI, N. \& MIANO, T.M., eds. Humic substances in the global environment and implications on human health. Amsterdam, Elsevier Science, 1994. p.115-120.

RICE, J .A. \& LIN,J .S. Fractal nature of humic materials. Environ. Sci. Tecnhol., 27:413-414,1993.

SENESI, N. The fractal approach to the study of humic substances. In: SENESI, N. \& MIANO, T.M., eds. Humic substances in the global environment and implications on human health. Amsterdam, Elsevier Science. 1994. p.3-41.

SENESI, N.; LOURUSOO, G.F.; MIANO, T.M.; MAGGIPINTO, G.; RIZZI, F.R. \& CAPOZZI, V. The fractal dimension of humic substances as function of $\mathrm{pH}$ by turbidity measurements. In: SENESI, N. \& MIANO, T.M., eds. Humic substances in the global environment and implications on human health. Amsterdam, Elsevier Science, 1994. p.121126.

SENESI, N.; RIZZI, F.R.; DELLINO, P. \& ACQUAFREDDA, P. Fractal dimension of humic acids in aquandous suspension as a function of $\mathrm{pH}$ and time. Soil Sci. Soc. Am. J ., 60:17731780,1996

SENESI, N.; RIZZI, F.R.; DELLINO, P. \& ACQUAFREDDA, P. Fractal humic acids in aqueous suspensions at various concentrations, ionic strengths, and pH values. Coll. Surf., 127:57-68, 1997.
SKOOG, D.A.; HOLLER, F.J . \& NIEMAN, T. A Principles of instrumental analysis. 5.ed. Philadel phia, Sauder Golden, 1992. 849p.

SPARK, K.M.; WELLS, J.D. \& J OHNSON, B.B. The interaction of a humic acid with heavy metals. Aust. J . Soil Res., 35:89101, 1997

STEVENSON, J .F. Humus chemistry - Genesis, composition, reactions 2.ed. New York, J ohn Wiley \& Sons, 1994. 496p.

SWIFT, R.S. Method for extraction of IHSS soil fulvic and humic acids. In: SPARK, K.M.; WELLS, J.D. \& J OHNSON, B.B Methods of soil analysis. Madison, Soil Science of Society of Americam, 1996. p.1018-1020.

SWIFT, R.S. Molecular weight, size, shape, and charge characteristics of humic substances: some basic considerations. In: HAYES, M.H.B., eds. Humic substance II: In search of structure. New York, J ohn Wiley \& Sons, 1989. p.449-465.

VICSEK, T. Fractal growth fenomena 2.ed. Singapore, World Scientic Publishing, 1992. 488p

WERSHAW, R.L. Sizes and shapes of humic substances by scattering techniques. In: HAYES, M.H.B.; MacCHARTY, P.; MALCOLM., R.L. \& SWIFT, R.S. Humic Substance II. New York, J ohn Wiley \& Sons, 1989. p.540-591. 